Voix et Images

voixetimages

\title{
Retours sur l'identité et la liberté
}

\section{Pascal Riendeau}

Volume 40, numéro 3 (120), printemps-été 2015

URI : https://id.erudit.org/iderudit/1032639ar

DOI : https://doi.org/10.7202/1032639ar

Aller au sommaire du numéro

Éditeur(s)

Université du Québec à Montréal

ISSN

0318-9201 (imprimé)

1705-933X (numérique)

Découvrir la revue

Citer ce compte rendu

Riendeau, P. (2015). Compte rendu de [Retours sur l'identité et la liberté]. Voix et Images, 40(3), 133-138. https://doi.org/10.7202/1032639ar

Ce document est protégé par la loi sur le droit d'auteur. L’utilisation des services d’Érudit (y compris la reproduction) est assujettie à sa politique d'utilisation que vous pouvez consulter en ligne.

https://apropos.erudit.org/fr/usagers/politique-dutilisation/
Cet article est diffusé et préservé par Érudit.

Érudit est un consortium interuniversitaire sans but lucratif composé de l’Université de Montréal, l’Université Laval et l’Université du Québec à Montréal. Il a pour mission la promotion et la valorisation de la recherche. https://www.erudit.org/fr/ 


\title{
ROM A N
}

\author{
Retours sur l'identité et la liberté

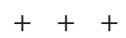

PASCAL RIENDEAU

Université de Toronto

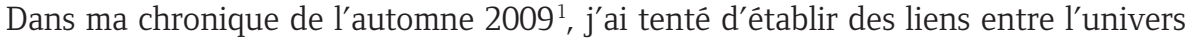
romanesque bien connu de Jacques Poulin et celui de Nicolas Dickner. J'insistais sur l'importance de quelques thématiques (notamment le roman de l'Amérique) ou sur le traitement de certains sujets (l'amitié entre un homme et une femme, par exemple). Grâce au hasard éditorial, ces deux auteurs peu prolifiques ont publié cette année un nouveau roman à environ un mois d'intervalle, comme un autre habitué de ces pages, Sergio Kokis. Fidèle à son habitude, Dickner a choisi, dans Six degrés de liberté2, de privilégier des personnages d'adolescents (qui deviendront de jeunes adultes à la fin du roman) dotés d'une intelligence supérieure, mais mal adaptés à leur environnement ou au monde en général. Élisabeth Routier-Savoie et Éric Le Blanc, qui ont quinze ans au début du récit, sont amis depuis la maternelle; "on les croyait différents, à tort: ils étaient complémentaires» (30). L'esprit géométrique d'Éric a trouvé en Lisa, "synthétique et narrative» (31), une alliée indispensable, surtout à partir du moment où il a commencé à souffrir sérieusement d'agoraphobie. Alors qu'il doit fréquenter l'école secondaire, Éric se retrouve confiné à sa chambre, réussissant à maintenir un lien avec les autres grâce à Lisa, qui l'aide à inventer différents projets scientifiques ou informatiques, surtout ce prototype de parachute muni d'un vieil appareil photo relié à un ordinateur personnel qui s'élève dans l'atmosphère afin de capter des images de la Terre. Dickner possède l'art d'exploiter les interactions adolescentes sans valoriser l'introspection. Leur amitié subit un coup dur quand Éric doit quitter son petit quartier résidentiel près de Huntingdon afin de suivre sa mère qui commence une nouvelle vie au Danemark. Petit génie de l'informatique, Éric fait fortune avec une rapidité exceptionnelle en investissant dans les conteneurs abandonnés - très nombreux après la crise économique de 2008 -, tandis que Lisa peine à continuer ses études collégiales, elle qui doit s'occuper d'un père malade. Tout cela change quand Lisa a soudain une idée, une illumination.

1 Pascal Riendeau, «De la nostalgie d'un monde possible à la possible fin du monde», Voix et Images, vol. XXXV, no 1, p. 120-125.

2 Nicolas Dickner, Six degrés de liberté, Québec, Alto, 2015, 384 p. 
Dickner crée un second récit qui, de prime abord, ne donne pas l'impression d'appartenir au même univers que le premier. Jay - dont on ne connaît pas le nom véritable - , ex-pirate informatique rattachée à une famille mexicaine redoutée, voit sa peine de prison pour vol d'identité commuée en une période de liberté surveillée au cours de laquelle elle s'engage à travailler pour la Gendarmerie royale du Canada (GRC). L'ennui de son emploi et la longueur du contrat l'incitent à s'intéresser à un mystérieux conteneur (surnommé Papa Zoulou) qui a quitté le port de Montréal en direction de Singapour, mais qui s'arrête dans différents ports, sans que l'on sache pourquoi il fait toutes ces escales et surtout ce qui se trouve à l'intérieur. Les services policiers pensent qu'il transporte des personnes, mais qui? Est-ce une nouvelle stratégie adoptée par un groupe terroriste? Jay n'y croit pas et choisit de mener une enquête parallèle. Seule, avec des moyens limités et sans autorisation, elle réussit néanmoins à traquer ceux qui font circuler sur les mers et océans du monde un conteneur insaisissable grâce à une chose simple: les déchets. Il s'agit là d'une problématique fréquente chez Dickner que l'on retrouve également dans la première chronique qu'il a publiée dans le journal Voir: «L'écrivain est le poisson-vidangeur de cet aquarium boueux que l'on nomme culture. Il filtre, il recycle, il brocante. Plus les déchets abondent, plus l'auteur a l'écaille lustrée. Un bon écrivain, en somme, devance toujours les éboueurs ${ }^{3}$.» Dans ses romans, la conception des déchets possède une signification beaucoup plus littérale: dans l'appartement modeste qu'habite Jay près de la station de métro Crémazie, tout provient des ordures. Ce sont justement les détritus que Lisa laisse dans l'entrepôt qu'elle a loué durant l'été afin de transformer son conteneur dans le dessein de le rendre adapté à la vie humaine qui permettent à Jay de retrouver sa trace et de reconstruire le récit des événements. En outre, c'est dans le bac de recyclage de Lisa qu'elle découvre les autres informations qui lui manquaient, notamment ce lien autrement impossible à établir entre elle et le jeune millionnaire danois qui a financé l'opération.

Que signifie vivre dans un conteneur? Difficile de le savoir, d'autant plus que le narrateur fournit peu de détails sur la vie de Lisa à bord. Cela nous mène vers une des problématiques principales soulevées par le roman : «comment narrer une histoire qui se déroule dans un lieu que personne ne peut conceptualiser?» (300) À l'aide du discours indirect libre, cette interrogation montre que ni le narrateur ni le personnage (Jay) n'imaginent la chose possible. En lisant un immense dossier sur les passagers clandestins, Jay cherche à comprendre l'histoire invraisemblable de Lisa, sans doute seule dans un conteneur, qui traverse les océans, reste brièvement en transit dans les grands ports de marchandise du monde, sans que l'on puisse jamais la repérer. Pourquoi aurait-elle entrepris ce voyage? Les six degrés du titre renvoient à cette conception scientifique (en génie ou en robotique) associée à la liberté d'un corps rigide, notamment celle d'un navire. Ce premier sens assez transparent en cache un second qui évoque une liberté paradoxale: le choix de Lisa de vivre enfermée dans un conteneur pendant des mois dans des conditions très éloignées de celles souvent insoutenables des migrants qui risquent leur vie en embarquant clandestinement

3 Nicolas Dickner, «Devancer les éboueurs» (2006), dans Le romancier portatif. 52 chroniques à emporter, Québec, Alto, 2011, p. 11. 
dans des conteneurs. Cette idée de Lisa peut lui être venue d'un souvenir de ce vieux magazine Life de 1962 qui se trouvait ouvert à la page d'un article intitulé «Six Degrees of Freedom» à l'intérieur d'une chambre secrète qu'elle découvre au cœur d'une grande maison que son père rénovait quelques années auparavant. Le projet d'Éric et Lisa - qu'on ne peut pas définir comme des militants - ne s'inscrit pas dans une dénonciation qui viserait les conditions excessivement dangereuses des migrants. Pour Lisa, il s'agit de pousser jusqu'à l'extrême l'expérience humaine à travers une exploration en solitaire de 20000 lieues sur les mers, mais qui se transforme en «duel à mort» (350) avec le temps, dont l'expérience ne peut plus vraiment être vécue et comprise comme une durée. Un autre élément suscite la fascination de Jay pour un tel projet, comme elle le laisse entendre à Éric quand elle le retrouve chez lui au Danemark: "un conteneur équipé pour traverser les murs... C'est mieux qu'une route. Mieux qu'un passeport. Avec ça, la géographie n'existe plus.» (362)

Dickner valorise la construction d'un récit complexe aux intrigues multiples auquel se greffe un réel suspense. Or ses romans, qui ne peuvent guère être qualifiés de philosophiques ni même d'essayistiques, ne négligent pas pour autant l'intégration de quelques idées plus stimulantes dans une trame narrative touffue. Si les réflexions paraissent parfois frivoles, on retrouve derrière la dérision un réel questionnement sur l'identité. C'est précisément la manière insolite de Dickner qui rend les idées plus romanesques. Qu'est-ce que les ordures disent de nous? Qu'est-ce que les autres peuvent apprendre en analysant ce que l'on jette? Horacio Guzman, ex-beau-père de Jay, chef du clan qu'elle a fréquenté durant sa période criminelle, lui a souvent fait la leçon sur l'art de se débarrasser correctement de ses ordures, en insistant sur leur cohérence: «Il orchestrait le contenu de ses sacs avec la minutie d'un artiste visuel qui aurait préparé une importante exposition au Guggenheim» (136), précise le narrateur. Il en est de même pour les identités virtuelles. Avant même de comprendre que l'énigmatique Éric Le Blanc contrôle et finance l'opération Papa Zoulou, Jay commence par retrouver sa mère grâce à son compte Facebook. Existe-t-il une bonne manière d'interpréter l'identité d'un individu à l'aide de Facebook? Qui est la bonne Isabelle Le Blanc? En composant un tableau à partir de toutes les données à sa disposition, Jay "a l'impression d'avoir mis les pieds dans un monde parallèle exclusivement peuplé d'Isabelle Le Blanc [...]. Qui est jeune, vieille, sans âge, porte le chapeau de paille et la minijupe. [...] Mais, surtout, Isabelle habite partout.» (184)

Le titre du quatorzième roman de Jacques Poulin, Un jukebox dans la tête ${ }^{4}$, compose une belle métaphore pour la mémoire affective du vieux Jack Waterman - alter ego de l'auteur - qu'évoquent chez lui les airs mélancoliques de chansons françaises, celles de Léo Ferré, d'Yves Montand, de Guy Béart, de Joe Dassin ou d'Henri Salvador. Après avoir cédé sa place à sa traductrice dans La traduction est une histoire d'amour ${ }^{5}$, puis à son petit frère, Francis, dans L'anglais n'est pas une langue

4 Jacques Poulin, Un jukebox dans la tête, Montréal, Leméac, 2015, 149 p.

5 Jacques Poulin, La traduction est une histoire d'amour, Montréal/Arles, Leméac/Actes Sud, 2006, 136 p. 
magique $^{6}$ et L'homme de la Saskatchewan ${ }^{7}$, Jack Waterman revient comme narrateur et protagoniste du roman pour la première fois depuis Chat sauvage ${ }^{8}$. Mais le rapprochement s'impose surtout avec Le vieux chagrin ${ }^{9}$, publié un quart de siècle plus tôt, même si le narrateur est en réalité l'écrivain Jim, avatar de Jack Waterman. On peut croire que tous les romans de Poulin se ressemblent - ce qui n'est pas entièrement faux -, mais la parenté entre Un jukebox dans la tête et Le vieux chagrin s'avère bien plus profonde que celle de quelques thèmes récurrents dans l'œuvre de l'auteur. Le vieux Jack malcommode et misanthrope des derniers romans donne l'impression d'avoir rajeuni et il rappelle davantage celui qui, dans Le vieux chagrin, accueille la Petite chez lui. Mélodie - pas encore trentenaire - est une jeune femme traquée qui trouve refuge chez l'écrivain, dont elle a lu toute l'œuvre. À la manière des Mille et une nuits, elle lui raconte en plusieurs soirs une histoire qui lui est arrivée alors qu'elle avait seize ans et qui revient la hanter. Elle est certaine qu'un homme la suit, la guette et qu'il vient d'emménager dans le même immeuble qu'elle. Mélodie possède tous les atouts pour séduire le vieil écrivain, mais n'est pas Schéhérazade qui veut. Son art de la narration reste parfois hésitant ou imprécis et nécessitera de l'aide. Elle compense ses carences de conteuse en mettant l'accent sur une charge émotive forte mais contenue et en cessant toujours assez brusquement son récit afin de maintenir le vieux Jack en haleine pour un autre épisode. L'alternance narrative qui s'impose rapidement rend le récit plus complexe et accentue la séduction entre les deux.

Bien qu'elle soit diffuse et qu'elle reste souvent très pudique, la sexualité constitue une partie essentielle de l'œuvre romanesque de Poulin. À seize ans, Mélodie, les «cheveux très courts» (56), possédait les mêmes caractéristiques physiques que la Petite du Vieux chagrin, victime de l'inceste d'un beau-père doux et charmeur. Jeune délinquante habituée à une vie plus rude, Mélodie décide quant à elle de défier celui qui l'a momentanément hébergée et fait preuve d'audace afin d'éviter un viol et de fuir son agresseur. Chez Poulin, le récit de la violence sexuelle à l'égard des jeunes filles se produit au moment où celle qui a été victime de harcèlement ou d'une agression maîtrise mieux les événements passés. L'histoire de Mélodie provoque des fantasmes érotiques chez Jack, ce qui n'étonnera pas les lecteurs du Vieux chagrin, dans lequel on retrouve des scènes ou des situations analogues (la proximité physique durant la conversation, le rapprochement dans la baignoire, l'érection impossible à dissimuler). Dans Un jukebox dans la tête, le vieux Jack conserve la même pudeur à l'égard de Mélodie, même si elle est maintenant une femme presque trentenaire: «Il m'était impossible de cacher le désir qui m'inspirait. Cependant, je ne pouvais pas donner libre cours à mes envies.» (128)

Le narrateur n'est pas qu'un homme rempli de compassion qui aide les femmes traquées ou en détresse. Il reste un auteur solitaire et atrabilaire qui réserve ses commentaires les plus grinçants pour le milieu littéraire et les auteurs de ce qu'il nomme

6 Jacques Poulin, L'anglais n'est pas une langue magique, Montréal/Arles, Leméac/Actes Sud, 2009, 160 p.

7 Jacques Poulin, L'homme de la Saskatchewan, Montréal/Arles, Leméac/Actes Sud, 2011, 125 p.

8 Jacques Poulin, Chat sauvage, Montréal/Arles, Leméac/Actes Sud, coll. «Babel», 2002 [1998], 191 p.

9 Jacques Poulin, Le vieux chagrin, Montréal/Arles, Leméac/Actes Sud, coll. «Babel», 1995 [1989], 190 p. 
la littérature-spectacle. Jack Waterman accorde un entretien à Jimmy, le narrateur du roman Les yeux bleus de Mistassini ${ }^{10}$, dans lequel il achève l'échange en lui lisant Les Dix Commandements de l'Écrivain. Cette fois-ci, il offre un contrepoint à cette liste très caustique en énumérant de manière ironique en huit points la façon de devenir une vedette littéraire, puis il ajoute un post-scriptum: «Une fois devenu vedette, tu écriras n'importe comment, les critiques ne diront rien.» (19) Poulin se répète, c'est indéniable, mais il demeure un romancier subtil qui aime donner à ses lecteurs plusieurs avenues interprétatives. Mine de rien est le nom de la chatte que Jack adopte à la fin du récit: «Un nom qui me fit sourire [...], car il correspondait à l'attitude qui avait été la mienne, depuis le jour de ma rencontre avec la belle Mélodie. Et peut-être même depuis toujours.» (147) Mine de rien - titre du dernier chapitre - se présente comme une autre piste que les «lecteurs fervents et assidus» (quatrième de couverture) peuvent suivre, en gardant en mémoire la dernière chanson à sortir du jukebox qui se déclenche dans la tête de Jack quand des sentiments puissants l'envahissent. Â la fin, précise-t-il, la chanson de Barbara, «Dis, quand reviendras-tu?» n'est pourtant provoquée par rien. Quand Jack Waterman reviendra-t-il?

En racontant à Mélodie une de ses nombreuses histoires de déplacements à travers la France, alors qu'il fuyait le bruit des voisins qui l'empêchait d'écrire, Jack Waterman mentionne son arrivée à Collioure, où est enterré le poète espagnol Antonio Machado, mort en exil, et il cite l'un de ses vers: «Toi qui marches, il n'y a pas de chemin» (66). Un extrait du même poème sert d'épigraphe au récit de Sergio Kokis, Le sortilège des chemins ${ }^{11}$. Il s'agit bien d'une heureuse coïncidence, car les univers des deux romanciers diffèrent totalement. Sergio Kokis livre un récit de vie, celui de la découverte de la randonnée pédestre à l'âge de soixante ans et de la transformation qui en a résulté. Les lecteurs d'Haruki Murakami ${ }^{12}$ reconnaîtront cette passion pour l'activité physique qui s'inscrit, chez le grand romancier japonais, dans une hygiène de vie. Comparée à la discipline que s'impose Murakami, l'entreprise de Kokis pourrait être qualifiée de plus détachée, d'assurément moins rigide, mais on retrouve la même envie de lier les activités d'endurance à la réflexion, à l'écriture et à la peinture. Le sortilège des chemins est construit comme le récit d'une conversion. L'auteur parle dans son préambule d'un album ${ }^{13}$ qui l'aurait inspiré, de sa nature peu sportive, de la première sortie très pénible de treize kilomètres à pied, de son désir d'abandonner rapidement le projet, de sa persistance malgré tout, puis de la satisfaction d'accomplir le périple de Saint-Jean-Pied-de-Port à Saint-Jacques-de-Compostelle, enfin de la nécessité de continuer, de reprendre la route, de parcourir de nombreux chemins

10 Jacques Poulin, Les yeux bleus de Mistassini, Montréal/Arles, Leméac/Actes Sud, 2002, 189 p.

11 Sergio Kokis, Le sortilège des chemins, avec six paysages de sentiers peints par l'auteur, Montréal, Lévesque éditeur, coll. «Réverbération», 2015, 194 p.

12 Haruki Murakami, Autoportrait de l'auteur en coureur de fond, traduit du japonais par Hélène Morita, Paris, Belfond, coll. «10/18», 2009 [2007], 192 p.

13 Bien que Kokis ne mentionne pas le titre de l'album, il s'agit probablement des Carnets de Saint-Jacquesde-Compostelle de François Dermaut, Grenoble, Glénat, 2003, 160 p. 
européens. Lucide, il précise d'emblée qu'il n'a reçu aucune inspiration divine, que l'expérience religieuse du pèlerinage à Compostelle lui importe peu. Il ira plus loin en ce sens à la fin de son premier voyage. La cause mystique, écrit Kokis,

me répugne surtout parce qu'il s'agit d'une explication extérieure, aliénante dans le sens étymologique du terme. En fait, le vrai pèlerin marche vers lui-même. Le poète Machado a raison: il n'y a pas de chemin, il y a seulement des marcheurs, des chemineaux de la vie. Et chacun marche vers son lieu de nostalgie, à la recherche de ce qui donnera un sens à son cheminement. (77)

On reconnaît le style de Kokis, mais on note aussi une plus grande simplicité du récit, inspiré en partie des carnets de voyage de sa femme, Isle. Dans ce récit initiatique et touristique, Kokis décrit les lieux, les conditions de marche, les repas, les visites du pays, mais s'arrête aussi en chemin pour amorcer des mouvements introspectifs, plus brefs et plus limités que dans L'amour du lointain ${ }^{14}$, qui faisait, dix ans plus tôt, le point sur son travail de romancier. Kokis insiste beaucoup sur les plaisirs de la table qui accompagnent les longues randonnées, mais surtout sur la joie des rencontres avec les autres marcheurs ou les habitants des villages où le couple s'arrête. On retrouve une candeur dans le récit de Kokis, lui qui avoue avoir voulu suspendre son jugement et décrire l'expérience d'une nouvelle manière d'être au monde. Outre une salutaire autocritique, Kokis n'hésite pas à glisser des commentaires sarcastiques sur Montréal, le Brésil, ou l'art contemporain, lieux communs auxquels il nous a habitués. Le récit a été écrit en 2014, soit dix ans après le premier pèlerinage, ce qui instaure une distance nécessaire pour mieux procéder à un retour sur soi. Dans le récit de Kokis, le travail du souvenir et l'interrogation identitaire sont constitués de découvertes et de reprises, alors que chez Poulin, la nostalgie et la mélancolie dominent. Quant au roman de Dickner, la conception de l'identité, plus floue et fragmentée, y maintient un lien essentiel avec la transmission et l'héritage.

14 L'amour du lointain. Récit en marge des textes, Montréal, XYZ éditeur, coll. «Romanichels», 2004, 309 p. 